\title{
Asymptotics of Solutions of Some Integral Equations Connected with Differential Systems with a Singularity
}

\author{
M. Yu. Ignatiev
}

Mikhail Yu. Ignatiev, https://orcid.org/0000-0002-4354-9197, Saratov State University, 83 Astrakhanskaya St., Saratov 410012, Russia, mikkieram@gmail.com, ignatievmu@info.sgu.ru

Our studies concern some aspects of scattering theory of the singular differential systems $y^{\prime}-x^{-1} A y-q(x) y=\rho B y, x>0$ with $n \times n$ matrices $A, B, q(x), x \in(0, \infty)$, where $A, B$ are constant and $\rho$ is a spectral parameter. We concentrate on investigation of certain Volterra integral equations with respect to tensor-valued functions. The solutions of these integral equations play a central role in construction of the so-called Weyl-type solutions for the original differential system. Actually, the integral equations provide a method for investigation of the analytical and asymptotical properties of the Weyl-type solutions while the classical methods fail because of the presence of the singularity. In the paper, we consider the important special case when $q$ is smooth and $q(0)=0$ and obtain the classical-type asymptotical expansions for the solutions of the considered integral equations as $\rho \rightarrow \infty$ with $o\left(\rho^{-1}\right)$ rate remainder estimate. The result allows one to obtain analogous asymptotics for the Weyl-type solutions that play in turn an important role in the inverse scattering theory.

Keywords: differential systems, singularity, integral equations, asymptotical expansions.

Received: 26.06.2019 / Accepted: 01.07.2019 / Published: 02.03.2020

This is an open access article distributed under the terms of Creative Commons Attribution License (CC-BY 4.0)

DOI: https://doi.org/10.18500/1816-9791-2020-20-1-17-28

\section{INTRODUCTION}

Our studies concern some aspects of scattering theory of the differential systems

$$
y^{\prime}-x^{-1} A y-q(x) y=\rho B y, \quad x>0
$$

with $n \times n$ matrices $A, B, q(x), x \in(0, \infty)$, where $A, B$ are constant and $\rho$ is a spectral parameter.

Differential equations with coefficients having non-integrable singularities at the end or inside the interval often appear in various areas of natural sciences and engineering. For $n=2$, there exists an extensive literature devoted to different aspects of spectral theory of the radial Dirac operators, see, for instance [1-5].

Systems of the form (1) with $n>2$ and arbitrary complex eigenvalues of the matrix $B$ appear to be considerably more difficult for investigation even in the "regular" case $A=0$ [6]. Some difficulties of principal matter also appear due to the presence of the singularity. Whereas the "regular" case $A=0$ has been studied fairly completely to date [6-8], for the system (1) with $A \neq 0$ there are no similar general results.

The important role in scattering theory is played by a certain distinguished basis of generalized eigenfunctions for (1) (the so-called Weyl-type solutions, see, for instance [9]). In the presence of the singularity construction and investigation of this basis encounters some difficulties which do not appear in the "regular" case $A=0$. In particular, one can not use the auxiliary Cauchy problems with the initial conditions 
at $x=0$. The approach presented in [10] (see also [11] and references therein) for the scalar differential operators

$$
\ell y=y^{(n)}+\sum_{j=0}^{n-2}\left(\frac{\nu_{j}}{x^{n-j}}+q_{j}(x)\right) y^{(j)}
$$

is based on using some special solutions of the equation $\ell y=\lambda y$ that also satisfy certain Volterra integral equations. This approach assumes some additional decay condition for the coefficients $q_{j}(x)$ as $x \rightarrow 0$, moreover, the required decay rate depends on eigenvalues of the matrix $A$. In this paper, we do not impose any additional restrictions of such a type. Instead, we use a modification of the approach first presented in [12] for the higher-order differential operators with regular coefficients on the whole line and recently adapted for differential systems of the form (1) on the semi-axis in [9].

In brief outline the approach can be described as follows. We consider some auxiliary systems with respect to the functions with values in the exterior algebra $\wedge \mathbb{C}^{n}$. Our study of these auxiliary systems centers on two families of their solutions that also satisfy some asymptotical conditions as $x \rightarrow 0$ and $x \rightarrow \infty$ respectively, and can be constructed as solutions of certain Volterra integral equations. As in [12] we call these distinguished tensor solutions the fundamental tensors. The main difference from the above-mentioned method used in [10] is that we use the integral equations to construct the fundamental tensors rather than the solutions for the original system. Since each of the fundamental tensors has minimal growth (as $x \rightarrow 0$ or $x \rightarrow \infty$ ) among solutions of the same auxiliary system, this step does not require any decay of $q(x)$ as $x \rightarrow 0$.

Construction and properties of the fundamental tensors were considered in details in our paper [9] provided that $q(\cdot)$ is absolutely continuous and both $q, q^{\prime}$ are integrable on the semi-axis $(0, \infty)$. In this paper, we consider the important special case $q(0)=0$ and obtain the classical-type asymptotical expansions for the fundamental tensors as $\rho \rightarrow \infty$ with $o\left(\rho^{-1}\right)$ rate remainder estimate.

\section{ASSUMPTIONS AND NOTATIONS. FORMULATIONS OF THE RESULTS}

We are to discuss first the unperturbed system:

$$
y^{\prime}-x^{-1} A y=\rho B y
$$

and its particular case corresponding to the value $\rho=1$ of the spectral parameter

$$
y^{\prime}-x^{-1} A y=B y
$$

but to complex (in general) values of $x$.

Assumption 1. Matrix $A$ is off-diagonal. The eigenvalues $\left\{\mu_{j}\right\}_{j=1}^{n}$ of the matrix $A$ are distinct and such that $\mu_{j}-\mu_{k} \notin \mathbb{Z}$ for $j \neq k$, moreover, $\operatorname{Re} \mu_{1}<\operatorname{Re} \mu_{2}<\cdots<\operatorname{Re} \mu_{n}$, $\operatorname{Re} \mu_{k} \neq 0, k=\overline{1, n}$.

Assumption 2. $B=\operatorname{diag}\left(b_{1}, \ldots, b_{n}\right)$, the entries $b_{1}, \ldots, b_{n}$ are nonzero distinct points on the complex plane such that $\sum_{j=1}^{n} b_{j}=0$ and such that any 3 points are noncolinear. where

Under Assumption 1 system (4) has the fundamental matrix $c(x)=\left(c_{1}(x), \ldots, c_{n}(x)\right)$,

$$
c_{k}(x)=x^{\mu_{k}} \hat{c}_{k}(x),
$$

$\operatorname{det} c(x) \equiv 1$ and all $\hat{c}_{k}(\cdot)$ are entire functions, $\hat{c}_{k}(0)=\mathfrak{h}_{k}, \mathfrak{h}_{k}$ is an eigenvector of the matrix $A$ corresponding to the eigenvalue $\mu_{k}$. We define $C_{k}(x, \rho):=c_{k}(\rho x), x \in(0, \infty)$, 
$\rho \in \mathbb{C}$. We note that the matrix $C(x, \rho)$ is a solution of the unperturbed system (3) (with respect to $x$ for the given spectral parameter $\rho$ ).

Let $\Sigma$ be the following union of lines through the origin in $\mathbb{C}$ :

$$
\Sigma=\bigcup_{(k, j): j \neq k}\left\{z: \operatorname{Re}\left(z b_{j}\right)=\operatorname{Re}\left(z b_{k}\right)\right\}
$$

By virtue of Assumption 2 for any $z \in \mathbb{C} \backslash \Sigma$ there exists the ordering $R_{1}, \ldots, R_{n}$ of the numbers $b_{1}, \ldots, b_{n}$ such that $\operatorname{Re}\left(R_{1} z\right)<\operatorname{Re}\left(R_{2} z\right) \cdots<\operatorname{Re}\left(R_{n} z\right)$. Let $\mathscr{S}$ be a sector $\left\{z=r \exp (i \gamma), r \in(0, \infty), \gamma \in\left(\gamma_{1}, \gamma_{2}\right)\right\}$ lying in $\mathbb{C} \backslash \Sigma$. Then [13] the system (4) has the fundamental matrix $e(x)=\left(e_{1}(x), \ldots, e_{n}(x)\right)$ which is analytic in $\mathscr{S}$, continuous in $\overline{\mathscr{S}} \backslash\{0\}$ and admits the asymptotics:

$$
e_{k}(x)=\mathrm{e}^{x R_{k}}\left(\mathfrak{f}_{k}+x^{-1} \eta_{k}(x)\right), \quad \eta_{k}(x)=O(1), \quad x \rightarrow \infty, \quad x \in \overline{\mathscr{S}},
$$

where $\left(\mathfrak{f}_{1}, \ldots, \mathfrak{f}_{n}\right)=\mathfrak{f}$ is a permutation matrix such that $\left(R_{1}, \ldots, R_{n}\right)=\left(b_{1}, \ldots, b_{n}\right) \mathfrak{f}$. We define $E(x, \rho):=e(\rho x)$.

Everywhere below we assume that the following additional condition is satisfied.

Condition 1. For all $k=\overline{2, n}$ the numbers

$$
\Delta_{k}^{0}:=\operatorname{det}\left(e_{1}(x), \ldots, e_{k-1}(x), c_{k}(x), \ldots, c_{n}(x)\right)
$$

are not equal to 0 .

Under Condition 1 the system (4) has the fundamental matrix $\psi^{0}(x)=\left(\psi_{1}^{0}(x), \ldots, \psi_{n}^{0}(x)\right)$ which is analytic in $\mathscr{S}$, continuous in $\overline{\mathscr{S}} \backslash\{0\}$ and admits the asymptotics:

$$
\psi_{k}^{0}(x t)=\exp \left(x t R_{k}\right)\left(\mathfrak{f}_{k}+o(1)\right), \quad t \rightarrow \infty, \quad x \in \mathscr{S}, \quad \psi_{k}^{0}(x)=O\left(x^{\mu_{k}}\right), \quad x \rightarrow 0 .
$$

We define $\Psi^{0}(x, \rho):=\psi^{0}(\rho x)$. As above, we note that the matrices $E(x, \rho), \Psi^{0}(x, \rho)$ solve (3).

In the sequel we use the following notations:

- $\left\{\mathfrak{e}_{k}\right\}_{k=1}^{n}$ is the standard basis in $\mathbb{C}^{n}$;

- $\mathscr{A}_{m}$ is the set of all ordered multi-indices $\alpha=\left(\alpha_{1}, \ldots, \alpha_{m}\right), \alpha_{1}<\alpha_{2}<\cdots<\alpha_{m}$, $\alpha_{j} \in\{1,2, \ldots, n\}$;

- for a sequence $\left\{u_{j}\right\}$ of vectors and a multi-index $\alpha=\left(\alpha_{1}, \ldots, \alpha_{m}\right)$ we define $u_{\alpha}:=u_{\alpha_{1}} \wedge \cdots \wedge u_{\alpha_{m}}$;

- for a numerical sequence $\left\{a_{j}\right\}$ and a multi-index $\alpha$ we define $a_{\alpha}:=\sum_{j \in \alpha} a_{j}$, $a^{\alpha}:=\prod_{j \in \alpha} a_{j}$

- for a multi-index $\alpha$ the symbol $\alpha^{\prime}$ denotes the ordered multi-index that complements $\alpha$ to $(1,2, \ldots, n)$;

- for $k=\overline{1, n}$ we denote

$$
\vec{a}_{k}:=\sum_{j=1}^{k} a_{j}, \quad \overleftarrow{a}_{k}:=\sum_{j=k}^{n} a_{j}, \quad \vec{a}^{k}:=\prod_{j=1}^{k} a_{j}, \quad \overleftarrow{a}^{k}:=\prod_{j=k}^{n} a_{j}
$$

We note that Assumptions 1, 2 imply, in particular, $\sum_{k=1}^{n} \mu_{k}=\sum_{k=1}^{n} R_{k}=0$ and therefore for any multi-index $\alpha$ one has $R_{\alpha^{\prime}}=-R_{\alpha}$ and $\mu_{\alpha^{\prime}}=-\mu_{\alpha}$; 
- the symbol $V^{(m)}$, where $V$ is $n \times n$ matrix, denotes the operator acting in $\wedge^{m} \mathbb{C}^{n}$ so that for any vectors $u_{1}, \ldots, u_{m}$ the following identity holds:

$$
V^{(m)}\left(u_{1} \wedge u_{2} \wedge \cdots \wedge u_{m}\right)=\sum_{j=1}^{m} u_{1} \wedge u_{2} \wedge \cdots \wedge u_{j-1} \wedge V u_{j} \wedge u_{j+1} \wedge \cdots \wedge u_{m}
$$

- if $h \in \wedge^{n} \mathbb{C}^{n}$ then $|h|$ is a number such that $h=|h| \mathfrak{e}_{1} \wedge \mathfrak{e}_{2} \wedge \cdots \wedge \mathfrak{e}_{n}$;

- for $h \in \wedge^{m} \mathbb{C}^{n}$ we set $\|h\|:=\sum_{\alpha \in \mathscr{A}_{m}}\left|h_{\alpha}\right|$, where $\left\{h_{\alpha}\right\}$ are the coefficients from the expansion $h=\sum_{\alpha \in \mathscr{A}_{m}} h_{\alpha} \mathfrak{e}_{\alpha}$.

We use the same notation $L_{p}(a, b)$ for all the spaces of the form $L_{p}((a, b), \mathscr{E})$, where $\mathscr{E}$ is a finite-dimensional space. The notation $C[a, b]$ for the spaces of continuous functions will be used in a similar way.

Everywhere below the symbol $\mathscr{S}$ denotes some (arbitrary) open sector with the vertex at the origin lying in $\mathbb{C} \backslash \Sigma$.

For each fixed $\rho \in \overline{\mathscr{S}} \backslash\{0\}=: \mathscr{S}^{\prime}$ we consider the following Volterra integral equations $(k=\overline{1, n})$ :

$$
\begin{gathered}
Y(x)=T_{k}^{0}(x, \rho)+\int_{0}^{x} G_{n-k+1}(x, t, \rho)\left(q^{(n-k+1)}(t) Y(t)\right) d t, \\
Y(x)=F_{k}^{0}(x, \rho)-\int_{x}^{\infty} G_{k}(x, t, \rho)\left(q^{(k)}(t) Y(t)\right) d t
\end{gathered}
$$

where

$$
\begin{gathered}
T_{k}^{0}(x, \rho):=C_{k}(x, \rho) \wedge \cdots \wedge C_{n}(x, \rho), \\
F_{k}^{0}(x, \rho):=E_{1}(x, \rho) \wedge \cdots \wedge E_{k}(x, \rho)=\Psi_{1}^{0}(x, \rho) \wedge \cdots \wedge \Psi_{k}^{0}(x, \rho)
\end{gathered}
$$

and $G_{m}(x, t, \rho)$ is an operator acting in $\wedge^{m} \mathbb{C}^{n}$ as follows:

$$
G_{m}(x, t, \rho) f=\sum_{\alpha \in \mathscr{A}_{m}} \sigma_{\alpha}\left|f \wedge C_{\alpha^{\prime}}(t, \rho)\right| C_{\alpha}(x, \rho) .
$$

Here and below $\sigma_{\alpha}:=\left|\mathfrak{h}_{\alpha} \wedge \mathfrak{h}_{\alpha^{\prime}}\right|$.

For any $\rho \in \mathscr{S}^{\prime}$ equations (5) and (6) were shown to have the unique solutions $T_{k}(x, \rho)$ and $F_{k}(x, \rho)$ respectively such that (see [9] for details):

$$
\begin{aligned}
& \left\|T_{k}(x, \rho)\right\| \leqslant M \begin{cases}\mid \rho x)^{\overleftarrow{\mu}_{k}} \mid, & |\rho x| \leqslant 1 \\
\exp \left(\rho x \overleftarrow{R}_{k}\right) \mid, & |\rho x|>1\end{cases} \\
& \left\|F_{k}(x, \rho)\right\| \leqslant M \begin{cases}\mid \rho x)^{\vec{\mu}_{k}} \mid, & |\rho x| \leqslant 1 \\
\exp \left(\rho x \vec{R}_{k}\right) \mid, & |\rho x|>1\end{cases}
\end{aligned}
$$

We call the functions $F_{k}(x, \rho), T_{k}(x, \rho)$ the fundamental tensors. Note that the fundamental tensors solve the auxiliary systems

$$
Y^{\prime}=Q^{(m)}(x, \rho) Y, \quad Q(x, \rho):=x^{-1} A+\rho B+q(x)
$$

with $m=k$ and $m=n-k+1$. 
We note that the tensors $\left\{E_{\alpha}(x, \rho)\right\}_{\alpha \in \mathscr{A}_{m}}$ form the fundamental system of solutions for the system (10) in the "unperturbed" case. Therefore, the following representation holds:

$$
T_{k}^{0}(x, \rho)=\sum_{\alpha \in \mathscr{A}_{n-k+1}} T_{k \alpha}^{0} E_{\alpha}(x, \rho)
$$

with $x$-independent coefficients $T_{k \alpha}^{0}$. Taking into account the special construction of the fundamental matrices $C(x, \rho), E(x, \rho)$ one can conclude that the coefficients $T_{k \alpha}^{0}$ do not depend on $\rho$ as well.

The $G_{m}(x, t, \rho)$ terms in equations (5), (6) are actually the Green operator functions for the nonhomogeneous systems:

$$
Y^{\prime}=Q^{(m)}(x, \rho) Y+f(x) .
$$

In order to construct them one can use variuos fundamental systems of solutions of the unperturbed system (3). In particular the following representations hold:

$$
G_{m}(x, t, \rho) f=\sum_{\alpha \in \mathscr{A}_{m}} \chi_{\alpha}\left|f \wedge \Psi_{\alpha^{\prime}}^{0}(t, \rho)\right| \Psi_{\alpha}^{0}(x, \rho)=\sum_{\alpha \in \mathscr{A}_{m}} \chi_{\alpha}\left|f \wedge E_{\alpha^{\prime}}(t, \rho)\right| E_{\alpha}(x, \rho) .
$$

Here and below $\chi_{\alpha}:=\left|\mathfrak{f}_{\alpha} \wedge \mathfrak{f}_{\alpha^{\prime}}\right|$.

In the paper, we study the asymptotical behavior of the fundamental tensors for $\rho \rightarrow \infty$. In [9] the following expansions were obtained:

$$
\begin{gathered}
T_{k}(x, \rho)=T_{k}^{0}(x, \rho)+O\left(\rho^{-\varepsilon} \exp \left(\rho x \overleftarrow{R}_{k}\right)\right), \quad \varepsilon \in(0,1) \\
F_{k}(x, \rho)=F_{k}^{0}(x, \rho)+O\left(\rho^{-1} \exp \left(\rho x \vec{R}_{k}\right)\right)
\end{gathered}
$$

for any fixed $x \in(0, \infty)$ and $\rho \rightarrow \infty, \rho \in \mathscr{S}^{\prime}$. We show that under the additional condition $q(0)=0$ more detailed expansion can be obtained.

Let $W_{0}(\xi)$ be the function defined as follows:

$$
W_{0}(\xi)=(1-|\xi|) \xi+|\xi|^{2}, \quad|\xi| \leqslant 1, \quad W_{0}(\xi):=\left(W_{0}\left(\xi^{-1}\right)\right)^{-1}, \quad|\xi|>1 .
$$

Notice that $W_{0}(\xi)$ is continuous in $\xi \in \mathbb{C}$, never vanishes for nonzero $\xi$ and admits the estimate:

$$
M_{1}|\xi| \leqslant\left|W_{0}(\xi)\right| \leqslant M_{2}|\xi|
$$

for all $\xi \in \mathbb{C}$. Moreover, we have $W_{0}(\xi)=1$ if $|\xi|=1$ and the asymptotics $W_{0}(\xi)=\xi(1+o(1))$ hold as $\xi \rightarrow 0$ and $\xi \rightarrow \infty$.

We introduce the following weight functions:

$$
W_{k}(\xi):= \begin{cases}W_{0}\left(\xi^{\mu_{k}}\right) \exp \left(R_{k} \xi\right), & |\xi| \leqslant 1 \\ \exp \left(R_{k} \xi\right), & |\xi|>1\end{cases}
$$

From the definition and the above-mentioned properties of $W_{0}(\cdot)$ it follows that the weight functions $W_{k}(\cdot), k=\overline{1, n}$ are all continuous in $\mathscr{S}^{\prime}$, never vanish and admit the asymptotics $W_{k}(\xi)=\xi^{\mu_{k}}(1+o(1))$ as $\xi \rightarrow 0$. We define

$$
\tilde{F}_{k}(x, \rho):=\left(\vec{W}^{k}(\rho x)\right)^{-1} F_{k}(x, \rho), \quad \tilde{T}_{k}(x, \rho):=\left(\overleftarrow{W}^{k}(\rho x)\right)^{-1} T_{k}(x, \rho) .
$$


Theorem 1. Suppose that $q(\cdot)$ is an absolutely continuous off-diagonal matrix function such that $q(0)=0$. Denote by $\hat{q}_{o}(\cdot)$ the off-diagonal matrix function such that $\left[B, \hat{q}_{o}(x)\right]=-q(x)$ for all $x>0$ (here $[\cdot, \cdot]$ denotes the matrix commutator). Define the diagonal matrix $d(x)=\operatorname{diag}\left(d_{1}(x), \ldots, d_{n}(x)\right)$, where

$$
d_{k}(x):=\int_{x}^{\infty} t^{-1}\left(\left[\hat{q}_{o}(t), A\right]\right)_{k k} d t
$$

and set $\hat{q}(x):=\hat{q}_{o}(x)+d(x)$.

Suppose that all the functions $q_{i j}(\cdot), q_{i j}^{\prime}(\cdot)$ and $\tilde{q}_{i j}(\cdot)$, where $\tilde{q}(x):=\hat{q}^{\prime}(x)+x^{-1}[\hat{q}(x), A]$ are from $X_{p}:=L_{1}(0, \infty) \cap L_{p}(0, \infty), p>2$.

Then for each fixed $x>0$ and $\rho \rightarrow \infty, \rho \in \mathscr{S}^{\prime}$ the following asymptotics hold:

$$
\begin{gathered}
\rho\left(\tilde{T}_{k}(x, \rho)-\tilde{T}_{k}^{0}(x, \rho)\right)=d_{0 k} \tilde{T}_{k}^{0}(x, \rho)+\sum_{\alpha, \beta \in \mathscr{A}_{n-k+1}} T_{k \beta}^{0} g_{k \alpha \beta}(x) \exp \left(\rho x\left(R_{\beta}-\overleftarrow{R}_{k}\right)\right) \mathfrak{f}_{\alpha}+o(1) \\
\rho\left(\tilde{F}_{k}(x, \rho)-\tilde{F}_{k}^{0}(x, \rho)\right)=\sum_{\alpha \in \mathscr{A}_{k}} f_{k \alpha}(x) \mathfrak{f}_{\alpha}+o(1)
\end{gathered}
$$

Here

$$
d_{0 k}=-\sigma_{\alpha^{*}(k)}\left|\left(d^{(n-k+1)}(0) \mathfrak{h}_{\alpha^{*}(k)}\right) \wedge \mathfrak{h}_{\left(\alpha^{*}(k)\right)^{\prime}}\right|,
$$

$\alpha^{*}(k):=(k, \ldots, n)$ and the coefficients in the representations are defined as follows:

$$
f_{k \alpha}(x)=\chi_{\alpha}\left|\left(\hat{q}^{(k)}(x) \mathfrak{f}_{\alpha_{*}(k)}\right) \wedge \mathfrak{f}_{\alpha^{\prime}}\right|
$$

for $\alpha \neq \alpha_{*}(k):=(1, \ldots, k)$,

$$
\begin{gathered}
f_{k, \alpha_{*}(k)}(x)=-\sum_{\alpha \in \mathscr{A}_{k}} \int_{x}^{\infty} \chi_{\alpha_{*}(k)}\left|\left(q^{(k)}(t) \mathfrak{f}_{\alpha}\right) \wedge \mathfrak{f}_{\alpha_{*}^{\prime}(k)}\right| \chi_{\alpha}\left|\left(\hat{q}^{(k)}(t) \mathfrak{f}_{\alpha_{*}(k)}\right) \wedge \mathfrak{f}_{\alpha^{\prime}}\right| d t \\
g_{k \alpha \beta}(x)=\chi_{\alpha}\left|\left(\hat{q}^{(n-k+1)}(x) \mathfrak{f}_{\beta}\right) \wedge \mathfrak{f}_{\alpha^{\prime}}\right|
\end{gathered}
$$

for $\beta \neq \alpha$,

$$
g_{k \beta \beta}(x)=\sum_{\alpha \in \mathscr{A}_{n-k+1}} \int_{0}^{x} \chi_{\beta}\left|\left(q^{(n-k+1)}(t) \mathfrak{f}_{\alpha}\right) \wedge \mathfrak{f}_{\beta^{\prime}}\right| \chi_{\alpha}\left|\left(\hat{q}^{(n-k+1)}(t) \mathfrak{f}_{\beta}\right) \wedge \mathfrak{f}_{\alpha^{\prime}}\right| d t .
$$

\section{PROOF OF THEOREM 1}

We consider in details the function $T_{k}(x, \rho)$, for the function $F_{k}(x, \rho)$ similar arguments are valid.

For the function $\hat{T}_{k}(x, \rho):=\tilde{T}_{k}(x, \rho)-\tilde{T}_{k}^{0}(x, \rho)$ we have the representation $\hat{T}_{k}(\cdot, \rho)=(I d-\mathscr{K}(\rho))^{-1} v_{k}(\cdot, \rho)$, where $\mathscr{K}(\rho)$ is an operator of the form:

$$
(\mathscr{K}(\rho) f)(x):=\int_{0}^{x} \mathscr{G}_{n-k+1}(x, t, \rho)\left(q^{(n-k+1)}(t) f(t)\right) d t
$$

acting in $L_{\infty}(0, T), T \in(0, \infty)$ is arbitrary. Here and below

$$
\mathscr{G}_{n-k+1}(x, t, \rho):=\frac{\overleftarrow{W}^{k}(\rho t)}{\overleftarrow{W}^{k}(\rho x)} G_{n-k+1}(x, t, \rho)
$$




$$
v_{k}(x, \rho)=\int_{0}^{x} \mathscr{G}_{n-k+1}(x, t, \rho)\left(q^{(n-k+1)}(t) \tilde{T}_{k}^{0}(t, \rho)\right) d t .
$$

Let us consider first the function $v_{k}(x, \rho)$. From the identity:

$$
\begin{gathered}
\rho\left(q^{(n-k+1)}(t) T_{k}^{0}(t, \rho)\right) \wedge E_{\alpha^{\prime}}(t, \rho)= \\
=\frac{d}{d t}\left(\left(\hat{q}^{(n-k+1)}(t) T_{k}^{0}(t, \rho)\right) \wedge E_{\alpha^{\prime}}(t, \rho)\right)-\left(\tilde{q}^{(n-k+1)}(t) T_{k}^{0}(t, \rho)\right) \wedge E_{\alpha^{\prime}}(t, \rho),
\end{gathered}
$$

where $\alpha \in \mathscr{A}_{n-k+1}$ is arbitrary it follows the relation:

$$
\begin{gathered}
\rho \int_{x_{0}}^{x} G_{n-k+1}(x, t, \rho)\left(q^{(n-k+1)}(t) T_{k}^{0}(t, \rho)\right) d t= \\
=\left.G_{n-k+1}(x, t, \rho)\left(\hat{q}^{(n-k+1)}(t) T_{k}^{0}(t, \rho)\right)\right|_{t=x_{0}} ^{t=x}-\int_{x_{0}}^{x} G_{n-k+1}(x, t, \rho)\left(\tilde{q}^{(n-k+1)}(t) T_{k}^{0}(t, \rho)\right) d t .
\end{gathered}
$$

Passing to the limits as $x_{0} \rightarrow 0$ and taking into account that $\hat{q}_{o}(0)=0$ we arrive at the relation:

$$
\begin{gathered}
\rho v_{k}(x, \rho)=d_{0 k} \tilde{T}_{k}^{0}(x, \rho)+\sum_{\alpha \in \mathscr{A}_{n-k+1}} \chi_{\alpha}\left|\left(\hat{q}^{(n-k+1)}(x) \tilde{T}_{k}^{0}(x, \rho)\right) \wedge E_{\alpha^{\prime}}(x, \rho)\right| E_{\alpha}(x, \rho)- \\
\int_{0}^{x} \mathscr{G}_{n-k+1}(x, t, \rho)\left(\tilde{q}^{(n-k+1)}(t) \tilde{T}_{k}^{0}(t, \rho)\right) d t .
\end{gathered}
$$

Since $\tilde{q}_{j j}=0, j=\overline{1, n}$, from (13) and [14] we obtain (in particular) the estimate:

$$
\left\|v_{k}(\cdot, \rho)\right\|_{B C[0, \infty)}=O\left(\rho^{-1}\right), \quad \rho \in \mathscr{S}^{\prime} .
$$

In what follows if $V=V(x, \rho)$ is some matrix function then $\tilde{V}$ denotes the matrix function $\tilde{V}(x, \rho):=V(x, \rho)(W(\rho x))^{-1}$, where $W=\operatorname{diag}\left(W_{1}, \ldots W_{n}\right)$. Since $\tilde{\Psi}^{0}(x, \rho)$ is continuous and bounded in $[0, \infty) \times \overline{\mathscr{S}}$ we have:

$$
\left\|\mathscr{G}_{n-k+1}(x, t, \rho)\right\| \leqslant M, \quad 0<t \leqslant x<\infty, \quad \rho \in \mathscr{S}^{\prime}
$$

with some absolute constant $M$.

Using the boundedness of $\mathscr{G}_{n-k+1}(x, t, \rho)$ one can obtain the estimate (see also the proof of [9, Theorem 3.1]):

$$
\left\|\mathscr{K}^{r}(\rho)\right\| \leqslant M_{0} \frac{M_{1}^{r}}{r !}\left(\int_{0}^{T}\|q(t)\| d t\right)^{r},
$$

where the norm $\left\|\mathscr{K}^{r}(\rho)\right\|$ assumes the norm of the operator acting in $L_{\infty}(0, T)$ for arbitrary $T>0$ and the constants $M_{0}, M_{1}$ do not depend on $T$. This yields the estimate $\left\|(I d-\mathscr{K}(\rho))^{-1}\right\|=O(1)$ uniformly in $\rho \in \mathscr{S}^{\prime}$. Thus (with taking into account (14)), we obtain the auxiliary prior estimate for $\hat{T}_{k}$ :

$$
\left\|\hat{T}_{k}(\cdot, \rho)\right\|_{L_{\infty}(0, T)}=O\left(\rho^{-1}\right), \rho \in \mathscr{S}^{\prime}
$$


for any $T>0$.

In order to make a more detailed study we represent the operator $\mathscr{K}(\rho)$ in the form $\mathscr{K}(\rho)=\mathscr{K}_{0}(\rho)+\mathscr{K}_{1}(\rho)$, where:

$$
\begin{gathered}
\mathscr{K}_{0}(\rho) f(x):= \\
=\theta^{+}(|\rho x|-1) \sum_{\alpha \in \mathscr{A}_{n-k+1}} \chi_{\alpha} \int_{|\rho|^{-1}}^{x} \exp \left(\rho(x-t)\left(R_{\alpha}-\overleftarrow{R}_{k}\right)\right)\left|\left(q^{(n-k+1)}(t) f(t)\right) \wedge \mathfrak{f}_{\alpha^{\prime}}\right| \mathfrak{f}_{\alpha} d t
\end{gathered}
$$

Here and below the symbols $\theta^{ \pm}(\cdot)$ denote the Heaviside step functions:

$$
\theta^{+}(\xi)=\left\{\begin{array}{ll}
0, & \xi \leqslant 0, \\
1, & \xi>0,
\end{array} \quad \theta^{-}(\xi)=\left\{\begin{array}{ll}
1, & \xi \leqslant 0 \\
0, & \xi>0
\end{array}=1-\theta^{+}(\xi)\right.\right.
$$

Lemma 1. Under the conditions of Theorem 1 one has the estimate $\left\|\mathscr{K}_{1}(\rho)\right\|=O\left(\rho^{-1}\right)$.

Proof. We split the operator as follows: $\mathscr{K}_{1}=\mathscr{K}_{0}^{(1)}+\mathscr{K}_{1}^{(1)}+\mathscr{K}_{2}^{(1)}$, where:

$$
\begin{gathered}
\left(\mathscr{K}_{0}^{(1)} f\right)(x)=\theta^{-}(|\rho x|-1) \int_{0}^{x} \mathscr{G}_{n-k+1}(x, t, \rho)\left(q^{(n-k+1)}(t) f(t)\right) d t, \\
\left(\mathscr{K}_{1}^{(1)} f\right)(x)=\theta^{+}(|\rho x|-1) \int_{0}^{|\rho|^{-1}} \mathscr{G}_{n-k+1}(x, t, \rho)\left(q^{(n-k+1)}(t) f(t)\right) d t .
\end{gathered}
$$

By virtue of (15) we have:

$$
\left\|\mathscr{K}_{1}^{(1)} f\right\| \leqslant M\|f\| \cdot \int_{0}^{|\rho|^{-1}}\|q(t)\| d t \leqslant M|\rho|^{-1}\|f\| \cdot\|q(\cdot)\|_{L_{\infty}(0, T)} .
$$

Proceeding in a similar way and taking into account that $\left(\mathscr{K}_{0}^{(1)} f\right)(x) \neq 0$ only if $|\rho x| \leqslant 1$ one can obtain the similar estimate for $\left\|\mathscr{K}_{0}^{(1)} f\right\|$. totics

Let us consider $\mathscr{K}_{2}^{(1)}$. Using the representation (9) for $G_{n-k+1}(x, t, \rho)$, the asymp-

$$
E_{\alpha}(x, \rho)=\exp \left(\rho x R_{\alpha}\right)\left(\mathfrak{f}_{\alpha}+O\left((\rho x)^{-1}\right)\right),
$$

which is uniform in $|\rho x| \geqslant 1$ and taking into account that $\operatorname{Re}\left(\rho(x-t)\left(R_{\alpha}-\overleftarrow{R}_{k}\right)\right) \leqslant 0$ for any $0 \leqslant t \leqslant x, \rho \in \mathscr{S}^{\prime}, \alpha \in \mathscr{A}_{n-k+1}$ we obtain the estimate:

$$
\begin{gathered}
\theta^{+}(|\rho x|-1) \theta^{+}(|\rho t|-1) \theta^{+}(x-t) \| \mathscr{G}_{n-k+1}(x, t, \rho)\left(q^{(n-k+1)}(t) f(t)\right)- \\
\sum_{\alpha \in \mathscr{A}_{n-k+1}} \chi_{\alpha} \exp \left(\rho(x-t)\left(R_{\alpha}-\overleftarrow{R}_{k}\right)\right)\left|\left(q^{(n-k+1)}(t) f(t)\right) \wedge \mathfrak{f}_{\alpha^{\prime}}\right| \mathfrak{f}_{\alpha}\left\|\leqslant \frac{M}{|\rho t|}\right\| q(t) \|
\end{gathered}
$$

with some absolute constant $M$. Since under the conditions of Theorem 1 $t^{-1} q(t) \in L_{1}(0, \infty)$ the estimate above yields

$$
\left\|\mathscr{K}_{2}^{(1)} f\right\| \leqslant M|\rho|^{-1}\|f\| \cdot \int_{0}^{\infty} t^{-1}\|q(t)\| d t
$$

and therefore $\left\|\mathscr{K}_{2}^{(1)}\right\|=O\left(\rho^{-1}\right)$. 
Lemma 2. Under the conditions of Theorem 1 one has the estimate $\left\|\mathscr{K}_{0}^{2}(\rho)\right\|=O\left(\rho^{-1}\right)$.

Proof. We have:

$$
\begin{gathered}
\left(\mathscr{K}_{0}^{2} f\right)(x)=\theta^{+}(|\rho x|-1) \sum_{\alpha \in \mathscr{A}_{n-k+1}} \int_{|\rho|^{-1}}^{x} \exp \left(\rho(x-t)\left(R_{\alpha}-\overleftarrow{R}_{k}\right)\right) \chi_{\alpha} \times \\
\times\left|\left(q^{(n-k+1)}(t)\left(\mathscr{K}_{0} f\right)(t)\right) \wedge \mathfrak{f}_{\alpha^{\prime}}\right| \mathfrak{f}_{\alpha} d t \\
\chi_{\alpha}\left|\left(q^{(n-k+1)}(t)\left(\mathscr{K}_{0} f\right)(t)\right) \wedge \mathfrak{f}_{\alpha^{\prime}}\right|=\theta^{+}(|\rho t|-1) \times \\
\times \sum_{\beta \in \mathscr{A}_{n-k+1}} \chi_{\beta} \int_{|\rho|^{-1}}^{t} \exp \left(\rho(t-\tau)\left(R_{\beta}-\overleftarrow{R}_{k}\right)\right)\left|\left(q^{(n-k+1)}(\tau) f(\tau)\right) \wedge \mathfrak{f}_{\beta^{\prime}}\right| Q_{\alpha \beta}(t) d \tau
\end{gathered}
$$

where $Q_{\alpha \beta}(t):=\chi_{\alpha}\left|\left(q^{(n-k+1)}(t) \mathfrak{f}_{\beta}\right) \wedge \mathfrak{f}_{\alpha^{\prime}}\right|$.

Thus, we can rewrite:

$$
\left(\mathscr{K}_{0}^{2} f\right)(x)=\theta^{+}(|\rho x|-1) \sum_{\alpha \beta \in \mathscr{A}_{n-k+1}} \int_{|\rho|^{-1}}^{x}\left|\left(q^{(n-k+1)}(\tau) f(\tau)\right) \wedge \mathfrak{f}_{\beta^{\prime}}\right| H_{\alpha \beta}(x, \tau, \rho) d \tau,
$$

where:

$$
H_{\alpha \beta}(x, \tau, \rho)=\int_{\tau}^{x} Q_{\alpha \beta}(t) \exp \left(\rho(x-t)\left(R_{\alpha}-\overleftarrow{R}_{k}\right)+\rho(t-\tau)\left(R_{\beta}-\overleftarrow{R}_{k}\right)\right) \mathfrak{f}_{\alpha} d t
$$

We notice again that $\operatorname{Re}\left(\rho(x-t)\left(R_{\alpha}-\overleftarrow{R}_{k}\right)+\rho(t-\tau)\left(R_{\beta}-\overleftarrow{R}_{k}\right)\right) \leqslant 0$ for any $0 \leqslant \tau \leqslant t \leqslant x, \rho \in \mathscr{S}^{\prime}, \alpha, \beta \in \mathscr{A}_{n-k+1}$. Moreover, under the conditions of Theorem 1 $Q_{\alpha \beta}(\cdot)$ are absolutely continuous and $Q_{\alpha \beta}(t) \equiv 0$ if $\alpha=\beta$. This yields the estimate

$$
\theta^{+}(|\rho \tau|-1) H_{\alpha \beta}(x, \tau, \rho)=O\left(\rho^{-1}\right),
$$

which is uniform in $0 \leqslant \tau \leqslant x, \rho \in \mathscr{S}^{\prime}$. The estimate implies the required assertion.

Proof of Theorem 1. We have $\hat{T}_{k}(\cdot, \rho)=v_{k}(\cdot, \rho)+\mathscr{K}(\rho) v_{k}(\cdot, \rho)+\mathscr{K}^{2}(\rho) \hat{T}_{k}(\cdot, \rho)$.

We note that

$$
\left(\mathscr{K}(\rho) \tilde{T}_{k}^{0}(\cdot, \rho)\right)(x)=\int_{0}^{x} \mathscr{G}_{n-k+1}(x, t, \rho)\left(q^{(n-k+1)}(t) \tilde{T}_{k}^{0}(t, \rho)\right)=v_{k}(x, \rho)=O\left(\rho^{-1}\right)
$$

uniformly for $\rho \in \mathscr{S}^{\prime}, x \in(0, T)$.

This, prior estimate (16), (14) and Lemmas 1, 2 yield:

$$
\hat{T}_{k}(\cdot, \rho)=v_{k}(\cdot, \rho)+\mathscr{K}_{0}(\rho) \omega_{k}(\cdot, \rho)+O\left(\rho^{-2}\right),
$$

where

$$
\begin{aligned}
\rho \omega_{k}(x, \rho)= & \sum_{\alpha \in \mathscr{A}_{n-k+1}} \chi_{\alpha}\left|\left(\hat{q}^{(n-k+1)}(x) \tilde{T}_{k}^{0}(x, \rho)\right) \wedge E_{\alpha^{\prime}}(x, \rho)\right| E_{\alpha}(x, \rho)- \\
& -\int_{0}^{x} \mathscr{G}_{n-k+1}(x, t, \rho)\left(\tilde{q}^{(n-k+1)}(t) \tilde{T}_{k}^{0}(t, \rho)\right) d t .
\end{aligned}
$$

and the $O(\cdot)$ term assumes an estimate in $L_{\infty}(0, T)$ norm. 
From [14, Theorem 1] and (18) we have:

$$
\rho \omega_{k}(x, \rho)=\sum_{\alpha \in \mathscr{A}_{n-k+1}} \chi_{\alpha}\left|\left(\hat{q}^{(n-k+1)}(x) \tilde{T}_{k}^{0}(x, \rho)\right) \wedge E_{\alpha^{\prime}}(x, \rho)\right| E_{\alpha}(x, \rho)+o(1),
$$

that yields:

$$
\begin{gathered}
\theta^{+}(|\rho t|-1) \rho \omega_{k}(t, \rho)= \\
=\theta^{+}(|\rho t|-1) \sum_{\alpha, \beta \in \mathscr{A}_{n-k+1}} T_{k \beta}^{0} \exp \left(\rho t\left(R_{\beta}-\overleftarrow{R}_{k}\right)\right) \hat{Q}_{\alpha \beta}(t) \mathfrak{f}_{\alpha}+\rho^{-1} \hat{\omega}_{k}(t, \rho)+o(1)
\end{gathered}
$$

where $\hat{Q}_{\alpha \beta}(t)=\chi_{\alpha}\left|\left(\hat{q}^{(n-k+1)}(t) \mathfrak{f}_{\beta}\right) \wedge \mathfrak{f}_{\alpha^{\prime}}\right|$, the $o(\cdot)$ term assumes an estimate in $L_{\infty}(0, T)$ norm and $t \hat{\omega}_{k}(t, \rho)$ is uniformly bounded in $\{|\rho t| \geqslant 1\}$.

Under the conditions of Theorem 1 we have $t^{-1} q(t) \in L_{1}(0, \infty)$. This yields $\mathscr{K}_{0}(\rho) \hat{\omega}_{k}(\cdot, \rho)=O(1)$ and thus from the representation above we obtain:

$$
\begin{aligned}
& \left(\mathscr{K}_{0}(\rho) \omega_{k}(\cdot, \rho)\right)(x)=\rho^{-1} \theta^{+}(|\rho x|-1) \sum_{\alpha, \beta, \gamma \in \mathscr{A}_{n-k+1}} \chi_{\gamma} T_{k \beta}^{0} \int_{|\rho|^{-1}}^{x} \exp \left(\rho(x-t)\left(R_{\gamma}-\overleftarrow{R}_{k}\right)+\right. \\
& \left.\quad+\rho t\left(R_{\beta}-\overleftarrow{R}_{k}\right)\right) \hat{Q}_{\alpha \beta}(t)\left|\left(q^{(n-k+1)}(t) \mathfrak{f}_{\alpha}\right) \wedge \mathfrak{f}_{\gamma^{\prime}}\right| \mathfrak{f}_{\gamma} d t+o\left(\rho^{-1}\right)=\rho^{-1} \theta^{+}(|\rho x|-1) \times \\
& \quad \times \sum_{\beta, \gamma \in \mathscr{A}_{n-k+1}} T_{k \beta}^{0} \int_{|\rho|^{-1}}^{x} \exp \left(\rho(x-t)\left(R_{\gamma}-\overleftarrow{R}_{k}\right)+\rho t\left(R_{\beta}-\overleftarrow{R}_{k}\right)\right) \tilde{Q}_{\gamma \beta}(t) \mathfrak{f}_{\gamma} d t+o\left(\rho^{-1}\right)
\end{aligned}
$$

where:

$$
\tilde{Q}_{\gamma \beta}(t):=\sum_{\alpha \in \mathscr{A}_{n-k+1}} Q_{\gamma \alpha}(t) \hat{Q}_{\alpha \beta}(t), \quad Q_{\gamma \alpha}(t)=\chi_{\gamma}\left|\left(q^{(n-k+1)}(t) \mathfrak{f}_{\alpha}\right) \wedge \mathfrak{f}_{\gamma^{\prime}}\right|
$$

and the $o(\cdot)$ term assumes an estimate in $L_{\infty}(0, T)$. Under the conditions of Theorem 1 the functions $Q_{\alpha \beta}$ и $\hat{Q}_{\alpha \beta}$ (for any pair of multi-indices $\alpha, \beta$ ) are absolutely continuous. Therefore, we have for $\gamma \neq \beta$ :

$$
\int_{|\rho|^{-1}}^{x} \exp \left(\rho(x-t)\left(R_{\gamma}-\overleftarrow{R}_{k}\right)+\rho t\left(R_{\beta}-\overleftarrow{R}_{k}\right)\right) \tilde{Q}_{\gamma \beta}(t) d t=O\left(\rho^{-1}\right)
$$

that yields:

$$
\begin{gathered}
\left(\mathscr{K}_{0}(\rho) \omega_{k}(q, \cdot, \rho)\right)(x)= \\
=\rho^{-1} \theta^{+}(|\rho x|-1) \sum_{\beta \in \mathscr{A}_{n-k+1}} T_{k \beta}^{0} \exp \left(\rho x\left(R_{\beta}-\overleftarrow{R}_{k}\right)\right) \int_{|\rho|^{-1}}^{x} \tilde{Q}_{\beta \beta}(t) d t \mathfrak{f}_{\beta}+o\left(\rho^{-1}\right)
\end{gathered}
$$

Substituting the obtained asymptotics to the representation (17) we arrive at:

$$
\begin{gathered}
\hat{T}_{k}(x, \rho)=v_{k}(x, \rho)+\rho^{-1} \theta^{+}(|\rho x|-1) \times \\
\times \sum_{\beta \in \mathscr{A}_{n-k+1}} T_{k \beta}^{0} \exp \left(\rho x\left(R_{\beta}-\overleftarrow{R}_{k}\right)\right) \int_{|\rho|^{-1}}^{x} \tilde{Q}_{\beta \beta}(t) d t \tilde{f}_{\beta}+o\left(\rho^{-1}\right)
\end{gathered}
$$


Here, as above, the $o(\cdot)$ term assumes an estimate in $L_{\infty}(0, T)$ norm. But all the terms in (19) are actually continuous with respect to $x \in\left(\left|\rho^{-1}\right|, T\right)$. This means that the expansion can be considered in point-wise sense as $\rho \rightarrow \infty$ while $x>0$ is arbitrary fixed.

Now we notice that

$$
\int_{|\rho|^{-1}}^{x} \tilde{Q}_{\beta \beta}(t) d t \rightarrow \int_{0}^{x} \tilde{Q}_{\beta \beta}(t) d t=g_{k \beta \beta}(x)
$$

as $\rho \rightarrow \infty$. Then we use the representation (13) for $v_{k}(x, \rho)$ and thus we obtain the required asymptotics.

Acknowledgements: This work was supported by the Russian Science Foundation (project No. 17-11-01193).

\section{References}

1. Brunnhuber R., Kostenko A., Teschl G. Singular Weyl-Titchmarsh-Kodaira theory for one-dimensional Dirac operators. Monatshefte für Mathematik, 2014, vol. 174, pp. 515547. DOI: https://doi.org/10.1007/s00605-013-0563-5

2. Albeverio S., Hryniv R., Mykytyuk Ya. Reconstruction of radial Dirac operators. J. Math. Phys., 2007, vol. 48, iss. 4, 043501, 14 p. DOI: https://doi.org/10.1063/1.2709847

3. Albeverio S., Hryniv R., Mykytyuk Ya. Reconstruction of radial Dirac and Schr'odinger operators from two spectra. J. Math. Anal. Appl., 2008, vol. 339, iss. 1, pp. 45-57. DOI: https://doi.org/10.1016/j.jmaa.2007.06.034

4. Serier F. Inverse Problems Inverse spectral problem for singular Ablowitz-Kaup-Newell-Segur operators on [0, 1]. Inverse Problems, 2006, vol. 22, no. 4, pp. 1457-1484. DOI: https://doi.org/10.1088/0266-5611/22/4/018

5. Gorbunov O. B., Shieh C.-T., Yurko V. A. Dirac system with a singularity in an interior point. Applicable Analysis, 2016, vol. 95, iss. 11, pp. 2397-2414. DOI: https://doi.org/10.1080/00036811.2015.1091069

6. Beals R., Coifman R. R. Scattering and inverse scattering for first order systems. Comm. Pure Appl. Math., 1984, vol. 37, iss. 1, pp. 39-90. DOI: https://doi.org/10.1002/cpa.3160370105

7. Zhou X. Direct and inverse scattering transforms with arbitrary spectral singularities. Comm. Pure Appl. Math. 1989, vol. 42, iss. 7, pp. 895-938. DOI: https://doi.org/10.1002/cpa.3160420702

8. Yurko V. A. Inverse spectral problems for differential systems on a finite interval. Results Math., 2005, vol. 48, iss. 3-4, pp. 371-386. DOI: https://doi.org/10.1007/BF03323374

9. Ignatyev M. Spectral analysis for differential systems with a singularity. Results Math., 2017, vol. 71, iss. 3-4, pp. 1531-1555. DOI: https://doi.org/10.1007/s00025-016-0605-0

10. Yurko V. A. On higher-order differential operators with a singular point. Inverse Problems, 1993, vol. 9, no. 4, pp. 495-502. DOI: https://doi.org/10.1088/0266-5611/9/4/004

11. Fedoseev A. E. Inverse problems for differential equations on the half-line having a singularity in an interior point. Tamkang Journal of Mathematics, 2011, vol. 42, no. 3, pp. 343-354. DOI: https://doi.org/10.5556/j.tkjm.42.2011.879

12. Beals R., Deift P., Tomei C. Direct and inverse scattering on the line. Providence, Rhod Island, American Mathematical Society, 1988. 209 p.

13. Sibuya Yu. Stokes phenomena. Bull. Amer. Math. Soc., 1977, vol. 83, no. 5, pp. 10751077.

14. Ignatiev $M$. Integral transforms connected with differential systems with a singularity. Tamkang Journal of Mathematics, 2019, vol. 50, no. 3, pp. 253-268. DOI: https://doi.org/10.5556/j.tkjm.50.2019.3353 


\title{
Cite this article as:
}

Ignatiev M. Yu. Asymptotics of Solutions of Some Integral Equations Connected with Differential Systems with a Singularity. Izv. Saratov Univ. (N. S.), Ser. Math. Mech. Inform., 2020, vol. 20, iss. 1, pp. 17-28. DOI: https://doi.org/10.18500/1816-9791-2020-20-1-17-28

\section{УДК 517.984}

\section{Асимптотики решений некоторых интегральных уравнений, связанных с дифференциальными системами с особенностью}

\author{
М. Ю. Игнатьев
}

Игнатьев Михаил Юрьевич, кандидат фризико-математических наук, доцент кафредры математической фризики и вычислительной математики, Саратовский национальный исследовательский государственный университет имени Н. Г. Чернышевского, Россия, 410012, г. Саратов, ул. Астраханская, д. 83, mikkieram@gmail.com, ignatievmu@info.sgu.ru

В работе изучаются некоторые аспекты теории рассеяния для сингулярных систем дифрференциальных уравнений $y^{\prime}-x^{-1} A y-q(x) y=\rho B y, x>0$ со спектральным параметром $\rho$, где $A, B, q(x), x \in(0, \infty)-n \times n$ матрицы, причем матрицы $A, B$ постоянны. Основным предметом исследования являются некоторые вольтерровские интегральные уравнения относительно тензорно-значных функций. Решения этих уравнений играют центральную роль в построении так называемых решений типа Вейля для исходной системы дифрференциальных уравнений. Поскольку классические методы при наличии особенности оказываются неприменимыми, изучение рассматриваемых интегральных уравнений становится в этом случае ключевым этапом исследования аналитических и асимптотических свойств решений типа Вейля. В данной работе мы рассматриваем важный частный случай, когда матрица-функция $q(\cdot)$ является гладкой и $q(0)=0$. В этом случае для решений рассматриваемых интегральных уравнений удается получить асимптотические разложения при $\rho \rightarrow \infty$ с оценкой остаточного члена $o\left(\rho^{-1}\right)$. Полученный результат позволяет получить асимптотики для решений типа Вейля, играющие, в свою очередь, важную роль при исследовании обратной задачи рассеяния.

Ключевые слова: дифрференциальные системы, особенности, интегральные уравнения, асимптотические разложения.

Поступила в редакцию: 26.06.2019 / Принята: 01.07.2019 / Опубликована: 02.03.2020

Статья опубликована на условиях лицензии Creative Commons Attribution License (CC-BY 4.0)

Благодарности. Работа выполнена при поддержке Российского научного фонда (проект № 17-11-01193).

\section{Образец для цитирования:}

Ignatiev M. Yu. Asymptotics of Solutions of Some Integral Equations Connected with Differential Systems with a Singularity [Игнатьев M. Ю. Асимптотики решений некоторых интегральных уравнений, связанных с дифференциальными системами с особенностью] // Изв. Сарат. ун-та. Нов. сер. Сер. Математика. Механика. Информатика. 2020. Т. 20, вып. 1. С. 17-28. DOI: https://doi.org/10.18500/1816-9791-2020-20-1-17-28 